\title{
Flore lactique de fromageries d'alpages suisses
}

\author{
par \\ R. MARET et T. SOZZI \\ Departement de Développement Technolugique \\ Société d'Assistance Technique pour Produits Nestlé S.A. \\ Lausanne
}

\section{INTRODUCTION}

Dans les hauts alpages du canton du Valais est fabriqué, durant l'été, un fromage gras typique à pâte mi-dure, fine et richement aromatique, destiné essentiellement à être consommé après fusion devant un four ouvert : le " fromage à raclette ».

L'évolution actuelle des modes de vie a malheureusement eu pour corollaire un certain abandon des exploitations laitières alpestres et une diminution de la production traditionnelle alors que la demande en fromage à raclette augmentait dans le même temps.

Il nous a paru intéressant d'entreprendre une série d'études taxonomiques et biochimiques sur les diverses espèces lactiques de la flore naturelle présente dans quelques fromageries de montagne où les pâtres utilisent encore une technologie tout à fait traditionnelle.

\section{MATERIEL ET METHODES}

\subsection{Les échantillons}

Trois séries d'échantillons ont été prélevées en des temps et des lieux différents.

La première série concerne des prélèvements effectués le matin à l'alpage de la Lettaz (Bourg-St-Pierre) et à l'alpage de Chermontane (Bagnes) le 25 août 1972, à savoir :

- Alpage de la Lettaz (alt. $1907 \mathrm{~m}$ )

- Alpage de Chermontane : (alt. $2300 \mathrm{~m}$ ) un échantillon de lait trait le soir précédent,

: un échantillon d'azi (lactosérum acide pour fabrication de séré).

un échantillon de lait frais du matin, 
un échantillon de caillé frais (type Bagnes), un échantillon de beurre.

La seconde série d'échantillons a été prélevée, le 6 juillet 1973, le matin à l'alpage de Flore et d'Aïre et dans les Mayens de la Biollaz (Conthey) :

- Alpage de Flore (alt. $1900 \mathrm{~m}$ )

- Alpage d'Aïre (alt. $1900 \mathrm{~m}$ )

- Mayens de la Biollaz : 4. un échantillon de sérum (fabrication du matin).

La troisième série fut prélevée le 19 juillet 1973 à l'alpage du Cour (Liddes, alt. $2200 \mathrm{~m}$ ) et concerne des échantillons de lait de fabrication (lait du jour et lait de la veille).

\subsection{Techniques microbiologiques}

(cf. Sozzi et Shepherd [1] ; Sozzi et Maret [2]).

Après dilution progressive en Ringer stérile, les échantillons étaient inoculés dans les milieux suivants :

a) Plate count Agar (Difco) $=$ PCA, pour numération des germes totaux. Incubation à $40^{\circ} \mathrm{C}$.

b) Plate count Agar (Difco) additionné de 10 p.100 de lait écrémé stérile $=$ PCAL. Ce milieu avait été choisi pour favoriser les streptocoques lactiques. Incubation à $30^{\circ} \mathrm{C}$ et $40^{\circ} \mathrm{C}$.

c) Mannitol salt Agar (Difco) $=$ MSA, pour les microcoques (Chapmann [3]). Incubation à $30^{\circ} \mathrm{C}$.

d) MRS (de Man et al. [4]) pour les lactobacilles. Incubation à $30^{\circ} \mathrm{C}$ et $40^{\circ} \mathrm{C}$.

e) Mycophil Agar (BBL) = MYCO, pour les levures et les moisissures. Incubation à $30^{\circ} \mathrm{C}$.

Après isolement à partir des différents milieux, les souches pures étaient conservées par lyophilisation.

\subsection{Identification et définition des caractères biochimiques des souches isolées}

Nous avons utilisé la même technique que celle décrite dans un travail précédent (Sozzi et Maret [2]).

Pour les fermentations sucrées et divers autres tests classiques nous avons utilisé le système API (Vixotab - La Balme-les-Grottes - 
France) pour lactobacilles. Nous avons modifié la spécification des caractères de 40 à 49 par rapport à ceux réellement testés sur les galeries (voir tab. 1). Nous avons donc examiné à part, en tubes à essais, les caractères 42,43 et 49 , ceci nous ayant été suggéré par Gélin et Philippe [5].

\section{TABLEAU 1}

Caractères biochimiques selon API test, avec notations différentes de 40 à 49

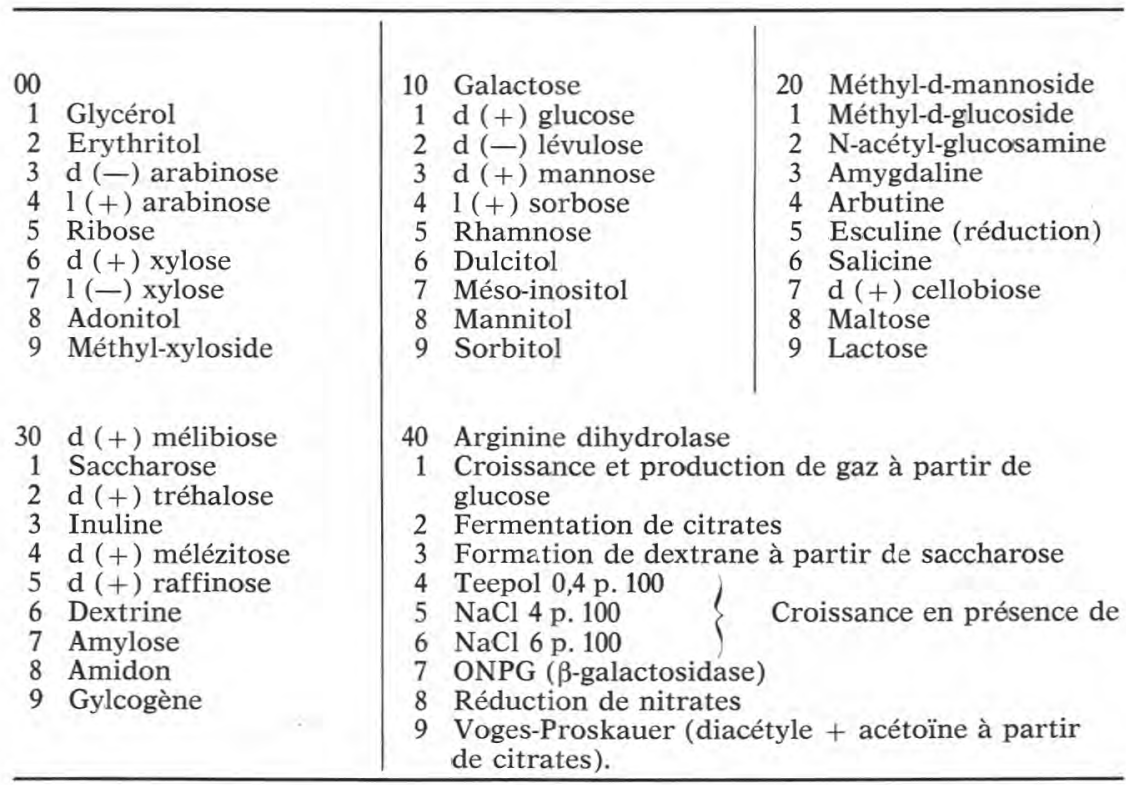

Les suspensions bactériennes utilisées pour les tests API avaient été préparées en MRS pour les lactobacilles et les leuconostocs et en Hogg et Jago [6] pour les streptocoques.

Après développement optimal, la biomasse était séparée par centrifugation et reprise dans le même milieu sans sucres contenant $2,5 \mathrm{~g} / 1$ de gélatine (ajoutée encore tiède), lavée plusieurs fois, puis utilisée en suspension de DO de 0,7 à 1,0 à $540 \mathrm{~nm}$ (cuvette de $1 \mathrm{~cm}$ ) pour la préparation des galeries d'identification.

\subsection{Température de croissance}

(cf. Sozzi et Maret [7]).

Les températures de croissance des micro-organismes ont été évaluées après croissance dans le milieu synthétique clair qui leur 
convenait le mieux. Les tubes inoculés ont été placés dans un appareil capable de réaliser un gradient de températures stable. L'intensité du trouble bactérien a été mesurée par évaluation de la densité à $540 \mathrm{~nm}$ après 7 et $24 \mathrm{~h}$.

\subsection{Propriétés. Caractères organoleptiques}

Les souches, après isolement, ont été ensemencées en lait complet stérile. Après incubation jusqu'à coagulation ou, au plus tard, jusqu'à $48 \mathrm{~h}$ pour les souches peu ou pas acidifiantes, les laits ont été dégustés.

\section{RESULTATS}

\subsection{Numération}

Nous n'avions pas évalué systématiquement le nombre de germes présents dans les échantillons de la première série. La seule valeur notée qui s'y rapporte est la suivante :

$6.10^{\circ}$ germes $/ \mathrm{ml}$ dans le lait frais de Chermontane, comptés sur MRS gélosé.

Les numérations effectuées sur les différents milieux des germes présents dans les échantillons des séries 2 et 3 sont présentées dans les tableaux 1 et 2 .

Les numérations sur MSA et MYCO concernent essentiellement les microcoques, les levures et quelques souches de Geotrichum candidum. Les résultats des études effectuées sur ces micro-organismes aérobies n'ont pas été consignés dans le présent travail.

On voit que tous les échantillons, le lait frais compris, sont assez riches en germes. Les prélèvements de lait frais avaient pourtant été réalisés de bonne heure le matin par une température relativement basse (altitude élevée).

\subsection{Caractères biochimiques des souches lactiques}

La définition des caractères biochimiques des souches isolées et leur identification ont été présentées dans les tableaux 4,5 et 6 . Nous n'avons présenté dans ce travail que les résultats concernant les bactéries lactiques typiques, c'est-à-dire les micro-aérophiles, catalase - négatives.

\subsubsection{LES STREPTOCOQUES}

Dans le cadre de ce travail, aucune souche de Streptococcus thermophilus n'a pu être isolée. 


\section{TABLEAU 2}

Nombre de germes/ml d'échantillons de la deuxième série, prélevés dans la région des alpages de Flore, d'Aïre et des Mayens de la Biollaz (Conthey, canton du Valais - Suisse)

\begin{tabular}{|c|c|c|c|c|c|c|c|}
\hline \multirow{2}{*}{$\begin{array}{c}\mathrm{N}^{\circ} \\
\text { échantillon }\end{array}$} & \multirow[b]{2}{*}{ PCA } & \multicolumn{2}{|c|}{ PCAL } & \multirow[b]{2}{*}{ MSA } & \multirow[b]{2}{*}{ MYCO } & \multicolumn{2}{|c|}{ MRS } \\
\hline & & $30^{\circ} \mathrm{C}$ & $40^{\circ} \mathrm{C}$ & & & $30^{\circ} \mathrm{C}$ & $40^{\circ} \mathrm{C}$ \\
\hline 1 a) & $67.10^{\circ}$ & $65.10^{6}$ & $58.10^{8}$ & $65.10^{5}$ & $20.10^{2}$ & $37.10^{6}$ & $64.10^{\circ}$ \\
\hline 2 & $3.10^{6}$ & ND b) & ND & $22.10^{4}$ & $19.10^{4}$ & $4.10^{6}$ & ND \\
\hline 3 & $23.10^{6}$ & $22.10^{6}$ & $8.10^{6}$ & 38 & $51.10^{2}$ & $20.10^{6}$ & $20.10^{6}$ \\
\hline 4 & $18.10^{7}$ & $18.10^{6}$ & $24.10^{6}$ & $14.10^{3}$ & ND & $23.10^{7}$ & $26.10^{\circ}$ \\
\hline
\end{tabular}

a) cf. 2.1.

b) Non déterminé.

TABLEAU 3

Nombre de germes/ml d'échantillons de la troisième série, prélevés à l'alpage du Cœur (Liddes, canton du Valais - Suisse)

\begin{tabular}{c|c|c|c|c|c|c|c}
\hline \multirow{2}{*}{$\begin{array}{c}\mathrm{N}^{\circ} \\
\text { échantillon }\end{array}$} & & \multicolumn{2}{|c|}{ PCAL } & & & \multicolumn{2}{c}{ MRS } \\
\cline { 3 - 7 } & PCA & $30^{\circ} \mathrm{C}$ & $40^{\circ} \mathrm{C}$ & MSA & MYCO & $30^{\circ} \mathrm{C}$ & $40^{\circ} \mathrm{C}$ \\
\hline 1 a) & $12.10^{7}$ & $46.10^{5}$ & $15.10^{5}$ & $<10^{3}$ & $44.10^{6}$ & $24.10^{5}$ & $17.10^{5}$ \\
\hline
\end{tabular}

a) cf. 2.1.

Les streptocoques trouvés dans les différents échantillons sont de type lactis (7 souches), faecium (3 souches) et faecalis (1 souche).

a) Les S. lactis

Caractères biochimiques : tableau 4 et figure 1.

Trois S. lactis ont été trouvés producteurs d'antibiotiques (probablement de type nisine). 
La souche VS $89 \mathrm{a}$, dans le lait, un net pouvoir épaississant. Ils ont des pouvoirs acidifiants variables.

b) Les $S$. faecium

Caractères biochimiques : tableau 4 et figure 2 .

Deux d'entre eux ont un faible pouvoir acidifiant.

c) Les $S$. faecalis

Caractères biochimiques : tableau 4 et figure 2 .

Ces micro-organismes acidifient lentement mais sont d'excellents producteurs d'arôme de type diacétyle.

\subsubsection{LES LEUCONOSTOCS}

Les leuconostocs ont été déterminés comme Leuconostoc cremoris (1 souche), L. lactis (3 souches), L. mesenteroides (2 souches).

Le tableau 5 et la figure 3 présentent quelques caractères biochimiques de ces souches.

Leur pouvoir acidifiant a été mesuré après une incubation de $24 \mathrm{~h}$.

\subsubsection{LES LACTOBACILLES}

Nous avons trouvé 3 souches de Lactobacillus casei casei, 1 souche de $L$. casei alactosus et 1 souche de L. brevis.

Le tableau 6 et la figure 4 présentent quelques caractères biochimiques de ces souches.

Leur pouvoir acidifiant a été mesuré après une incubation de 24 h.

\subsection{Température de croissance}

La figure 5 présente les courbes de croissance d'un S. lactis (5 A), d'un S. faecium (5 B), d'un Leuconostoc lactis (5 C) et d'un Lactobacillus casei (5 D) à diverses températures et en fonction du temps.

\subsection{Les propriétés organoleptiques}

A part quelques souches de Streptococcus lactis qui produisent des goûts étranges, dans l'ensemble, les micro-organismes isolés sont d'excellents producteurs d'arômes de type diacétyl-beurre.

\section{DISCUSSION}

Malgré la haute altitude et la fraîcheur des nuits, la flore lactique naturelle du lait était très abondante. Elle était constituée de Streptococcus de type lactis, faecalis, faecium et de Leuconostoc. Aucun Lactobacillus n'a été isolé des laits examinés. 


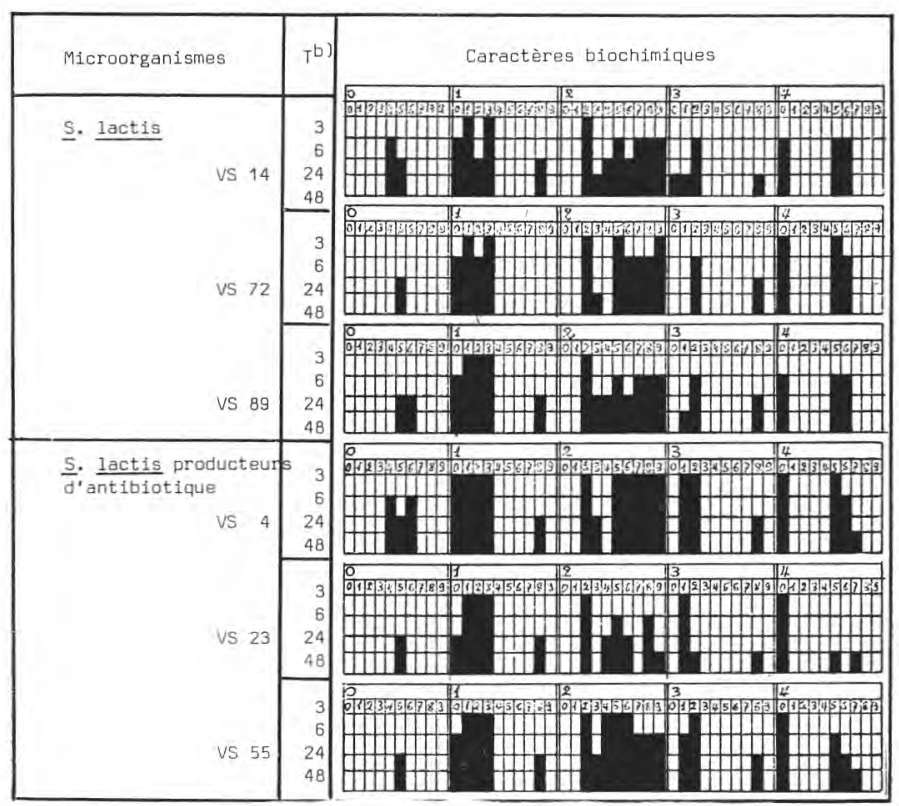

a) La spécification des caractères tels que notés de 40 à 49 a été modifiée selon le tableau 1.

b) $T$ = temps d'observation : après $3,6,24$ et 48 h d'incubation.

fig. 1

Profil d'apparition chronologique des caractères biochimiques observés sur API test a) des $S$. lactis isolés en divers alpages du canton du Valais (Suisse).

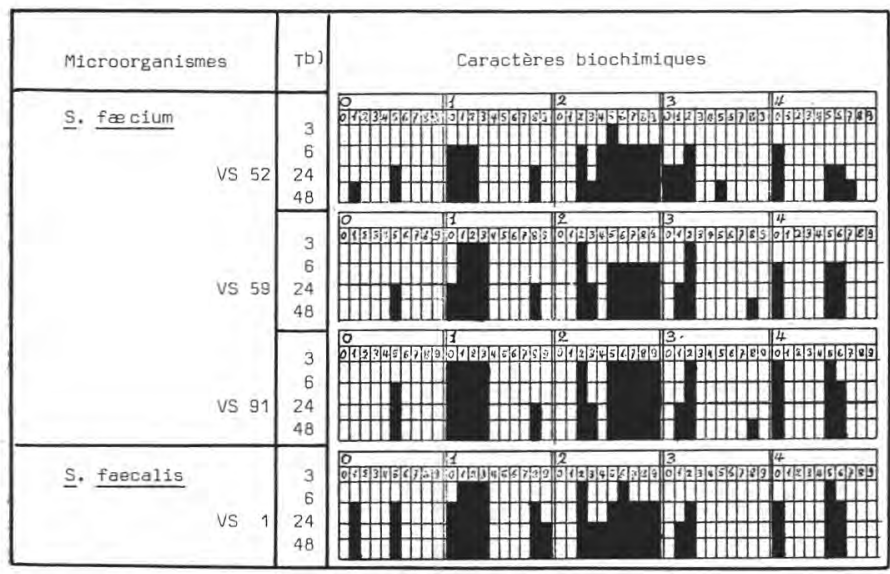

a) La spécification des caractères tels que notés de 40 à 49 a été modifiée selon le tableau 1.

b) $T$ = temps d'observation : après 3, 6, 24 et 48 h d'incubation.

fig. 2

Profil d'apparition chronologique des caractères biochimiques des $S$. faecium et d'un $S$. faecalis isolés en divers alpages du canton du Valais (Suisse). 
TABLEAU 4

Caractères biochimiques des streptocoques isolés en divers alpages du canton du Valais (Suisse)

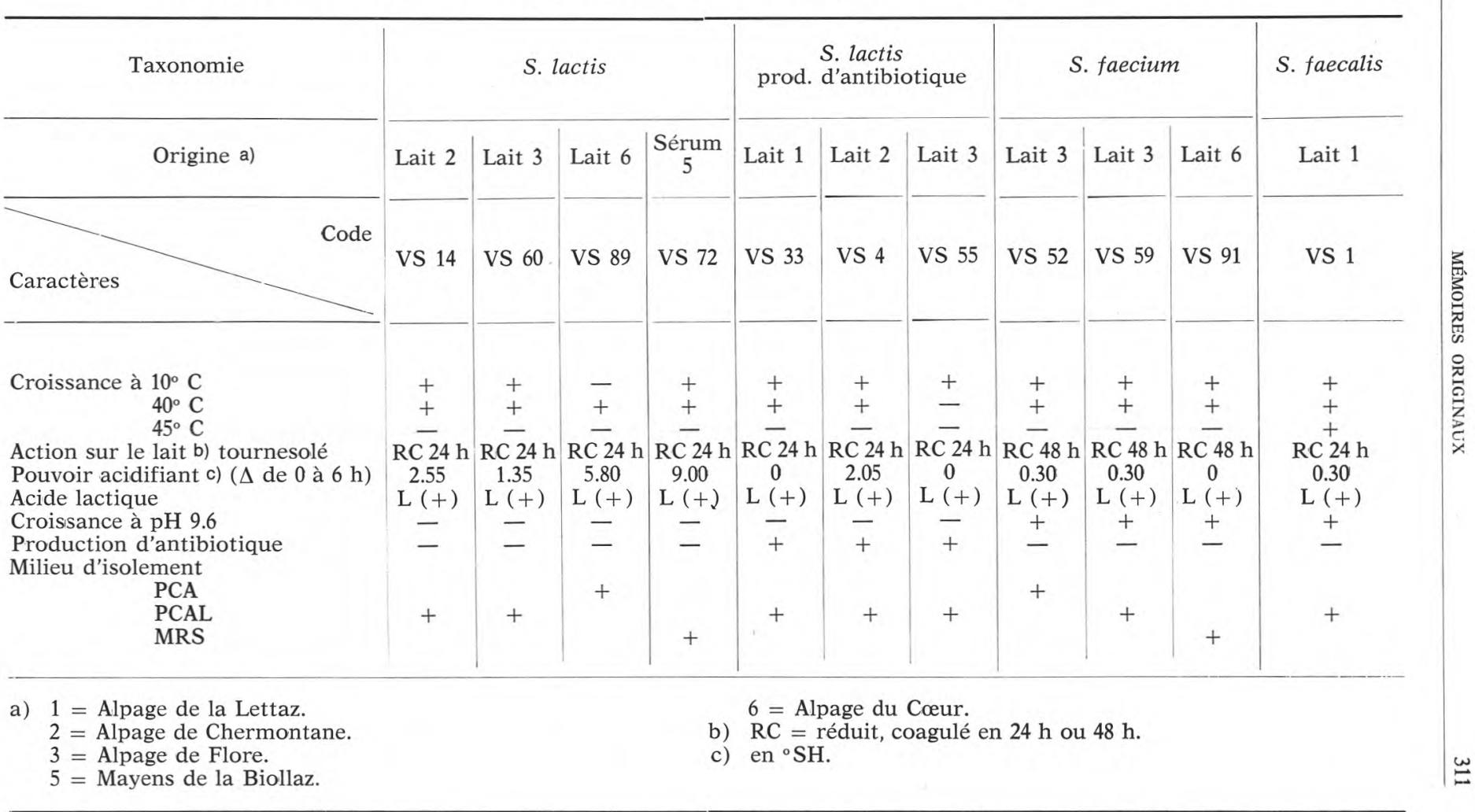


TABLEAU 5

Caractères biochimiques des leuconostocs isolés en divers alpages du canton du Valais (Suisse)

\begin{tabular}{|c|c|c|c|c|c|c|}
\hline \multirow{2}{*}{$\begin{array}{l}\text { Taxonomie } \\
\text { Origine a) }\end{array}$} & \multirow{2}{*}{$\begin{array}{l}\text { b) L. cremoris } \\
\text { Groupe I Garvie } \\
\text { Sérum } 3\end{array}$} & \multicolumn{3}{|c|}{$\begin{array}{l}\text { L. lactis } \\
\text { Groupe II Garvie }\end{array}$} & \multirow{2}{*}{$\begin{array}{c}\text { L. mesenteroides } \\
\text { Groupe III Garvie } \\
\text { Beurre } 2\end{array}$} & \multirow{2}{*}{$\begin{array}{c}\text { L. mesenteroides } \\
\text { Groupe V Garvie } \\
\text { Lait } 6\end{array}$} \\
\hline & & Lait 2 & Caillé 2 & Lait 6 & & \\
\hline Caractères & VS 61 & VS 23 & VS 26 & VS 92 & VS 48 & VS 99 \\
\hline $\begin{array}{c}\text { Production de } \mathrm{CO}_{2} \\
\text { Croissance à } 10^{\circ} \mathrm{C} \\
40^{\circ} \mathrm{C} \\
45^{\circ} \mathrm{C} \\
\text { Action sur le lait c) tournesolé } \\
\text { Pouvoir acidifiant d) }(\Delta \text { de } 0 \text { à } 24 \mathrm{~h}) \\
\text { Configuration de l'acide lactique } \\
\text { Milieux d'isolement : } \\
\text { PCA } \\
\text { PCAL } \\
\text { MRS }\end{array}$ & $\begin{array}{l}+ \\
+ \\
\overline{-} \\
\bar{I} \\
0 \\
D_{(-)} \\
+\end{array}$ & $\begin{array}{c}+ \\
+ \\
+ \\
\frac{-}{\operatorname{RC} 24 h} \\
13.40 \\
\mathrm{D}(-)\end{array}$ & $\begin{array}{c}+ \\
+ \\
+ \\
- \\
\operatorname{RC} 48 \mathrm{~h} \\
5.50 \\
\mathrm{D}(-) \\
\\
+\end{array}$ & $\begin{array}{c}+ \\
+ \\
+ \\
\frac{+}{24} \mathrm{~h} \\
2.60 \\
\mathrm{D}(-)\end{array}$ & $\begin{array}{c}+ \\
+ \\
+ \\
\text { C } 24 \mathrm{~h} \\
5.20 \\
\mathrm{D}(-)\end{array}$ & $\begin{array}{l}+ \\
+ \\
+ \\
\frac{\mathrm{I}}{} \\
0 \\
\mathrm{D}(-)\end{array}$ \\
\hline \multicolumn{2}{|l|}{ 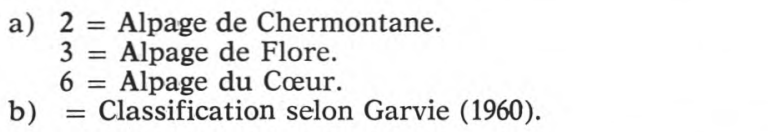 } & \multicolumn{3}{|c|}{$\begin{array}{l}\text { c) } \mathrm{RC}=\text { ré } \\
\mathrm{I}=\text { inch } \\
\text { d) en }{ }^{\circ} \mathrm{SH} .\end{array}$} & lé ; $C=$ coagulé, & éduit ; \\
\hline
\end{tabular}




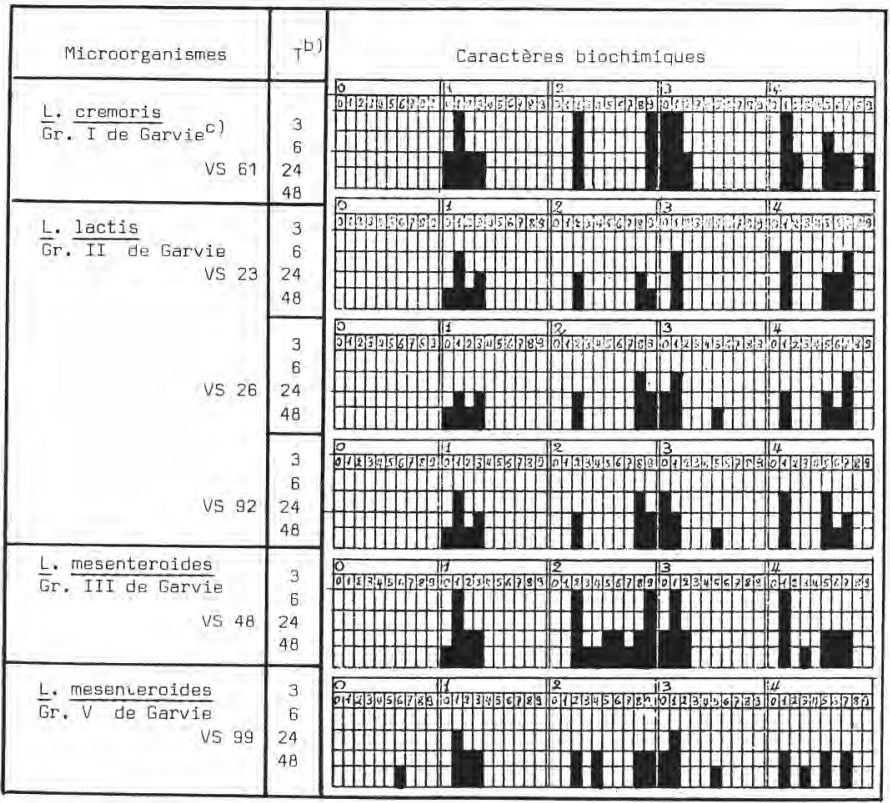

a) La spécífication des caractères tels que notés de 40 à 49 a été morifiée selon le tableau 1.

b) $T$ = temps d'observation : après $3,5,24$ et $48 \mathrm{~h}$ d'incubation.

c) Garvie, 1967

fig. 3

Profil d'apparition chronologique des caractères biochimiques observés sur API test a) des Leuconostocs isolés en divers alpages du canton du Valais (Suisse).

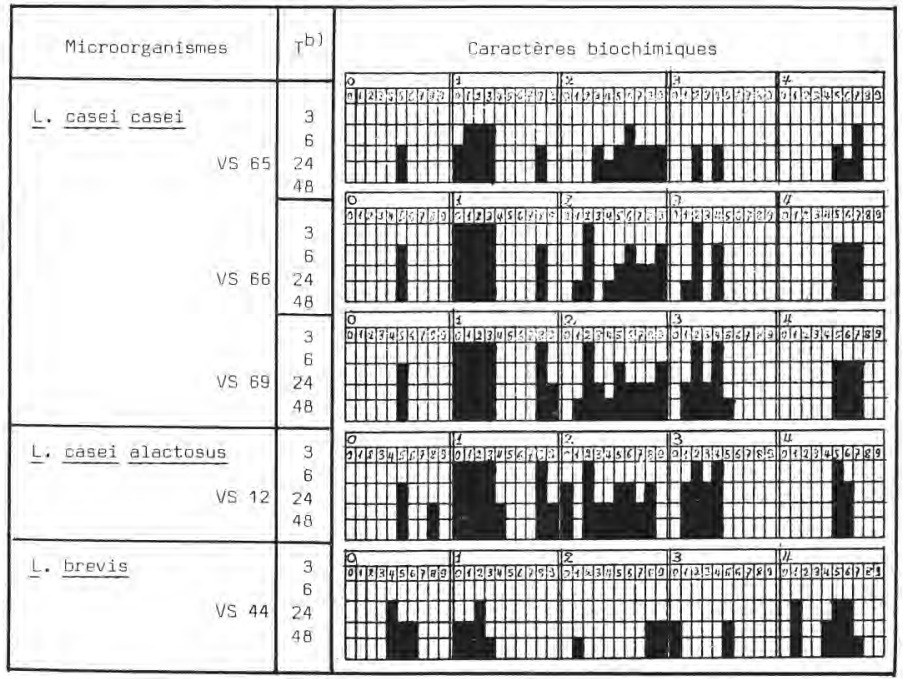

a) La spécification des caractères tels que notés de 40 à 49 a été modifiće selon le tableau 1.

b) $T$ = temps d'observation : aprềs $3,6,24$ et 48 h d'incubation.

fig. 4

Profil d'apparition chronologique des caractères biochimiques observés sur API test a) des lactobacilles isolés en divers alpages du canton du Valais (Suisse). 
TABLEAU 6

Caractères biochimiques des lactobacilles isolés en divers alpages du canton du Valais (Suisse)

\begin{tabular}{|c|c|c|c|c|c|}
\hline Taxonomie & \multicolumn{3}{|c|}{ L. casei casei } & L. casei alactosus & L. brevis \\
\hline Origine a) & Azi 4 & Azi 4 & Azi 4 & Azi 1 & Azi 1 \\
\hline Caractères & VS 65 & VS 66 & VS 69 & VS 12 & VS 44 \\
\hline $\begin{array}{l}\text { Production de } \mathrm{CO}_{2} \\
\text { Croissance à } 15^{\circ} \mathrm{C} \\
45^{\circ} \mathrm{C} \\
\text { Action sur le lait b) tournesolé } \\
\text { Pouvoir acidifiant c) ( } \Delta \text { de } 0 \text { à } 24 \mathrm{~h}) \\
\text { Acide lactique } \\
\text { Milieu d'isolement : } \\
\text { PCA } \\
\text { MRS }\end{array}$ & $\begin{array}{c}- \\
+ \\
+ \\
\mathrm{RC} 24 \mathrm{~h} \\
3.10 \\
\mathrm{~L}(+) \\
+\end{array}$ & $\begin{array}{c}- \\
+ \\
+ \\
\mathrm{RC} 24 \mathrm{~h} \\
3.20 \\
\mathrm{~L}(+) \\
+\end{array}$ & $\begin{array}{c}- \\
+ \\
+ \\
\operatorname{RC} 24 \mathrm{~h} \\
4.70 \\
\mathrm{~L}(+) \\
\\
+\end{array}$ & $\begin{array}{l}- \\
+ \\
+ \\
\text { I } 48 \mathrm{~h} \\
0 \\
\mathrm{~L}(+) \\
+\end{array}$ & $\begin{array}{l}+ \\
+ \\
+ \\
\text { I } 48 \mathrm{~h} \\
0 \\
\mathrm{DL}(+) \\
+\end{array}$ \\
\hline
\end{tabular}

a) 1 = Alpage de la Lettaz. 4 = Alpage d'Aïre.

b) $\mathrm{RC} 24 \mathrm{~h}=$ réduit, coagulé en $24 \mathrm{~h}$; I $48 \mathrm{~h}=$ inchangé après $48 \mathrm{~h}$.

c) ${ }^{\circ}{ }^{\circ} \mathrm{SH}$ 

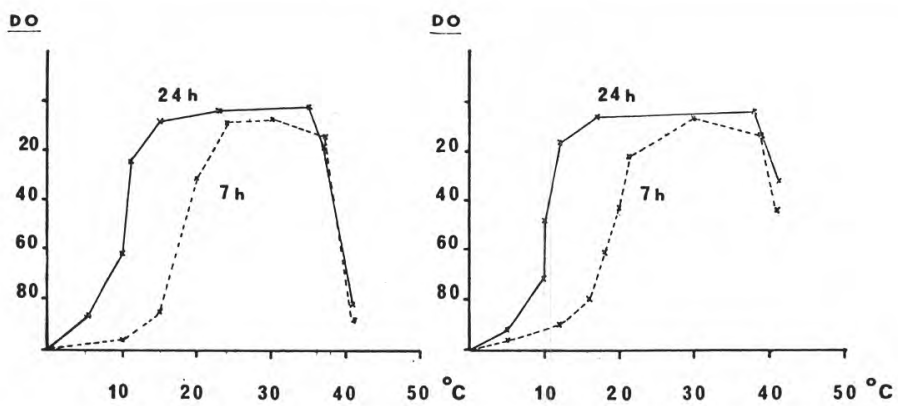

A :

Str lactis Vs 89

B :

Str faecium Vs 59

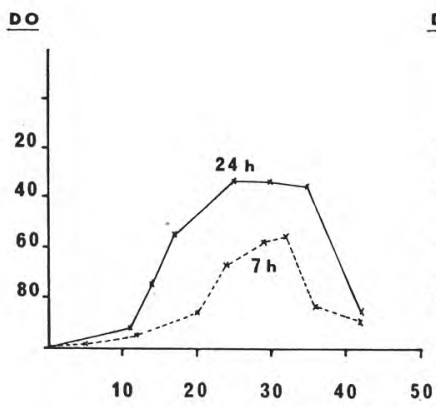

Do

C: Leuconostoc lactis Vs 23

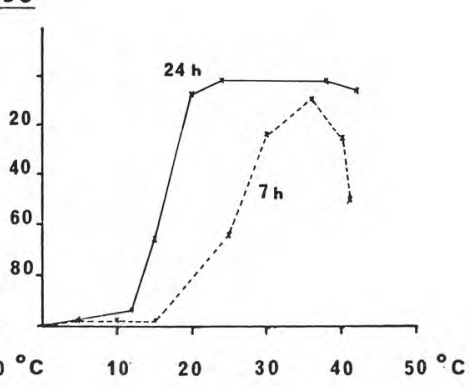

D: $\quad+$ casei $V_{5} 65$

fig. 5

Développement en fonction du temps à diverses températures du S. lactis VS 89 (A), du S. faecium VS 59 (B), du Leuconostoc lactis VS 23 (C) et du Lactobacillus casei VS 65 (D.).

La flore des sérums a été trouvée semblable à celle du lait. Le seul échantillon de beurre étudié a été trouvé colonisé par une abondante population de leuconostocs.

Les lactobacilles mésophiles sont la flore dominante des azis (sérum acide). Les homofermentaires de type casei y voisinent avec des hétérofermentaires de type brevis.

La présence de Streptococcus lactis producteurs d'antibiotiques (de type nisine) a été mise en évidence dans trois laits de fabrication.

Le pouvoir acidifiant des $S$. lactis est très variable. 
D'une façon générale l'utilisation des API tests s'est révélée très utile pour l'identification des micro-organismes. En raison de l'étendue des caractères testés simultanément et de la bonne reproductibilité de la méthode, il a été possible d'éliminer de nombreuses souches semblables isolées d'un même échantillon. En effet, si l'on examine les mêmes caractères en tubes à essais, les fluctuations sont beaucoup plus importantes en raison des différences possibles dans la composition du milieu ou dans l'allure générale du développement des souches. Avec le système API, les risques de considérer, comme différentes, des souches isolées d'un même clone, sont beaucoup plus faibles. La situation taxonomique des composantes écologiques de tel ou tel échantillon apparaît avec beaucoup plus de netteté.

Mais il est évident que le système API - qui permet également d'évaluer rapidement les traits dominants de la population globale d'un échantillon - ne suffit pas à caractériser telle ou telle souche si l'on veut en faire une utilisation technologique. Il conviendra, dans ce cas, de réaliser une série d'examens complémentaires : pouvoirs aromatique, acidifiant, protéolytique ; production d'antibiotiques, lysotypie, etc.

Nous avons réalisé, sur quelques souches, un test de croissance à différentes températures et en fonction de la durée d'incubation. Il en ressort que la possibilité de développement de l'ensemble des souches examinées augmente en fonction du temps d'incubation vers les basses températures. Les streptocoques, après 24 h, se développent encore nettement à des températures inférieures à $10^{\circ} \mathrm{C}$. Le leuconostoc et le lactobacille $L$. casei poussent plus difficilement aux basses températures. Ce test est important lorsqu'on veut utiliser correctement un micro-organisme pour une maturation fromagère donnée. Il serait préférable de choisir des souches capables de se développer - surtout dans le cas d'une flore complémentaire aromatisante - à la température de maturation du produit considéré. Or, nous savons par expérience, qu'à ce point de vue, des souches d'espèce identique ne se comportent pas de la même façon.

\section{CONCLUSIONS}

La flore naturelle lactique de laits prélevés dans divers alpages du canton du Valais destinés à la fabrication d'un fromage gras de montagne utilisé essentiellement pour la raclette a été étudiée de même que celle d'échantillons de sérum, d'azi et de beurre prélevés dans le même environnement technologique.

Les laits, les sérums et le beurre étaient colonisés par une flore mésophile de streptocoques et de leuconostocs. Le petit-lait acide ou 
azi contenait une flore de lactobacilles homo et hétérofermentaires mésophiles.

La présence de souches de Streptococcus lactis productrices d'antibiotiques a été relevée dans trois laits : la dominance occasionnelle de tels micro-organismes - heureusement faiblement acidifiants dans le cas présent - dans ces laits destinés à une production fromagère pourrait entraîner une raréfaction momentanée ou un mauvais développement des autres espèces.

L'absence des lactobacilles dans les laits est normale car ils colonisent plutôt l'espace technologique lui-même - ustensiles, récipients, air ambiant de la fromagerie - et ne se développent que plus tardivement. Ils peuvent ainsi, en raison de leur petit nombre dans le lait, ne pas apparaître lors des isolements. La croissance préalable des streptocoques les stimule toujours et ils peuvent proliférer plus tard dans les fromages, après un temps de latence plus ou moins important. Une étude complémentaire se devrait donc d'examiner l'évolution d'une telle flore au cours de la maturation fromagère.

La présente étude suggère également en quel sens on pourrait agir si l'on se proposait de recréer ou d'enrichir une flore naturelle par des souches actives destinées à assurer une fabrication correcte dans un ensemble technico-écologique éloigné le moins possible de la réalité traditionnelle.

\section{Remerciements}

Nous remercions Mme B. Marchesini et $M$. O. Depierraz de leur collaboration technique.

\section{R és u m é}

Pour le présent travail ont été étudiées les flores lactiques naturelles du lait, du lactosérum, du petit-lait acide (appelé " azi ") et du beurre de plusieurs alpages du canton du Valais (Suisse), produisant un fromage à pâte mi-dure, fine et richement aromatique, nommé « fromage à raclette».

Alors que le lait, le sérum et le beurre ne contiennent que des streptocoques et des leuconostocs, la flore de l' " azi » est composée de lactobacilles homo et hétérofermentaires. Toutes les souches isolées sont mésophiles.

L'absence de lactobacilles dans les laits a été discutée, de même ont été abordés les problèmes posés par l'industrialisation de la production de ce type de fromage, ainsi que la question des souches productrices d'antibiotiques. 


\section{S u $\mathbf{m} \mathbf{m}$ a $\mathbf{r} \mathbf{y}$}

The natural lactic flora encountered in milk, whey, acid whey (named " azi ") and butter from several mountain pastures ( alpages ") of the Canton of Valais (Switzerland), which produces a semihard, fine and very aromatic cheese named "fromage à raclette ", was studied.

While milk, whey and butter contain only streptococci and leuconostocs, the flora of " azi » is composed of homo and heterofermentative lactobacilli. All strains which have been isolated are mesophilic.

The absence of lactobacilli in the milks, the problems involved in the industrial manufacture of this type of cheese, and the question of antibiotic-producting strains are discussed.

Reçu pour publication en décembre 1975.

\section{Bibliographie}

[1] Sozzi (I.), SHePherd (D.) (1972). - Evolution de la composition chimique et de la flore microbienne du fromage Vacherin au cours de la maturation. Le Lait, 52, 203-219.

[2] Sozzi (T.), Maret (R.) (1973). - Etude sur la microflore lactique du fromage "Vacherin Mont-d'Or ». Le Lait, 53, 280-294.

[3] Chapman (G. H.) (1945). - The significance sodium chloride in studies of Staphylococci. J. Bact., 50, 201.

[4] De Mann (J. C.), Rogosa (M.) and Sharpe (E.) (1960). - A medium for the cultivation of Lactobacilli. J. Appl. Bact., 23, 130.

[5] Gelin (M.), PhilipPe (G.) (1974). - Communication personnelle.

[6] HoG (Mc. C. D.), JAGO (G. R.) (1970). - Extraction of the $260 \mathrm{~nm}$ absorbing material from group N. Streptococci as a method for estimating all growth. J. Dairy Res., 37, 199-202.

[7] Sozzi (T.), MARET (R.) (1975). - Isolement et quelques caractéristiques des bactériophages de Streptococcus thermophilus et de Lactobacillus helveticus de ferments d'Emmental. Le Lait, 55, 269-288. 